\title{
Asymptotics of the contour of the stationary phase and efficient evaluation of the Mellin-Barnes integral for the $F_{3}$ structure function
}

\author{
Aleksander V. Sidorov, ${ }^{1, *}$ Vasil I. Lashkevich, ${ }^{2}$ and Olga P. Solovtsova ${ }^{1,2, \dagger}$ \\ ${ }^{1}$ Bogoliubov Laboratory of Theoretical Physics, Joint Institute for Nuclear Research, \\ Dubna, Moscow Region, 141980 Russia \\ ${ }^{2}$ International Center for Advanced Studies, Gomel State Technical University, \\ Prospect Octiabria, 48, Gomel, 246746 Belarus
}

(Received 7 December 2017; published 19 April 2018)

\begin{abstract}
A new approximation is proposed for the contour of the stationary phase of the Mellin-Barnes integrals in the case of its finite asymptotic behavior as $\operatorname{Re} z \rightarrow-\infty$. The efficiency of application of the proposed contour and the quadratic approximation to the contour of the stationary phase is compared to the example of the inverse Mellin transform for the structure function $F_{3}$. It is shown that, although for a small number of terms $N$ in quadrature formulas used to calculate integrals along these contours, the quadratic contour is more efficient, but for $N>20$ the asymptotic stationary phase integration contour gives better accuracy. The case of the $Q^{2}$ dependence of the $F_{3}$ structure function is also considered.
\end{abstract}

DOI: 10.1103/PhysRevD.97.076009

\section{INTRODUCTION}

The Mellin-Barnes (MB) integrals [1] are widely used at high-energy physics. Recently, significant progress has been made in the numerical computation of these integrals. The review of digital packages is presented in the introduction to the paper [2]. The list of physical tasks solved using the MB integrals (two-loop massive Bhabha scattering in QED, in three-loop massless form factors and static potentials, in massive two-loop QCD form factors, in B-physics studies, in hadronic top-quark physics, and for angular integrations in phase-space integrals) can be supplemented by the problem of determining the structure functions and parton distributions in $x$ space on the basis of their Mellin moments.

The inverse Mellin transform method is widely used in calculations related to deep inelastic scattering (DIS) [3-5] for describing the scaling violation in polarized and unpolarized structure functions and fragmentation functions. The general expression for the inverse Mellin transform is written as a contour integral in the complex $z$ plane in the form

$$
f(x)=\frac{1}{2 \pi i} \int_{C} d z x^{-z} \tilde{f}(z)
$$

\footnotetext{
sidorov@theor.jinr.ru

olsol@theor.jinr.ru
}

Published by the American Physical Society under the terms of the Creative Commons Attribution 4.0 International license. Further distribution of this work must maintain attribution to the author(s) and the published article's title, journal citation, and DOI. Funded by SCOAP . where the contour $C$ usually runs parallel to the imaginary axis, to the right of the rightmost pole. In the case of the DIS, a function $\tilde{f}$ on the right-hand side of expression (1) is the moments of the structure function at some fixed value of momentum transfer squared $Q_{0}^{2}$ and is usually expressed in terms of the ratio of $\Gamma$ functions, such as the expression (27) for the Mellin moments of the $F_{3}$-structure function, which will be investigated in Sec. IV. Then, the integral (1) can be considered as a typical one-dimensional MB integral.

The best efficiency in a numerical integration Eq. (1) can be achieved on the contour of the stationary phase, where the oscillations of the integrand are minimal. However, the solution of the differential equation for the stationary phase contour and its subsequent application to calculate the MB integral requires big computing expenses (see, e.g., Ref. [6]). Instead, it is proposed to build such approximations of the stationary phase contour that would allow the effective application of the quadrature integration formulas [2]. It can be said that the first attempt to construct an effective approximation for the contour using the expansion of the integrand at the saddle point was made by Kosower [7] as applied to the calculation of parton distributions in the $x$ space. We call this contour $C_{K}$. Recently, it was proposed to construct the Padé approximation of the stationary phase contour, taking into account the presence of a saddle point in the integrand and its asymptotic behavior for large $z$ [2]. The effectiveness of this approach is shown in the summary table [2]. However, according to this table, for the integrand $F_{1}(z, s)=(-s)^{-z} \Gamma^{3}(-z) \Gamma(1+z) / \Gamma(-2 z)$ at $s=-1 / 20$ the relative accuracy $10^{-8}$ is achieved by taking into account 16 polynomials in the quadrature formula, whereas for the contour $C_{K}$, we achieve the same accuracy 
with 12 terms. In this concrete case, the use of the integration contour $C_{K}$ is more effective than the use of the Padé approximation of the stationary phase contour constructed in Ref. [2]. When does the contour $C_{K}$, which does not take into account the asymptotic behavior, allow us to obtain a reasonable result? Will the advantages of this contour be preserved with increasing the required accuracy? The answer to these questions will prompt our consideration in this work.

Here, we propose a new approximation for the contour of the stationary phase. The asymptotic behavior of the constructed contour coincides with the contour of the stationary phase as $\operatorname{Re} z \rightarrow-\infty$. We consider a particular case of a finite asymptotic behavior of the contour of the stationary phase in the limit $\operatorname{Re} z \rightarrow-\infty$. The MB integral arising for the $F_{3}$ structure function corresponds to this case. First, the construction of the contour will be presented on a simpler example of the MB integral whose value is known exactly. The corresponding integral arising in Feynman diagrams was examined, for example, in Ref. [2], where it was denoted by $I_{5}(s)$. Further, we consider in detail the application of the new asymptotic contour in the calculation of the $F_{3}$ structure function on the basis of their Mellin moments, and give a comparison with the results of applying the integration contour $C_{K}$.

It should be stressed that the choice of a contour of integration is dictated by a physical task. For example, in the calculation of massive diagrams it is usually enough to calculate the $\mathrm{MB}$ integral once but with a high relative accuracy of $\sim 10^{-10}-10^{-16}$. In the case of finding the shape of the structure functions, the accuracy of $\sim 10^{-4}-10^{-5}$ is sufficient for the fit of experimental data. However, in the process of fitting, the integral has to be calculated many times (more than $10^{6}$ times), so the computational speed that directly depends on the number of terms in the quadrature formula $N$ is important. It is known that the application of the contour proposed by Kosower is effective for a small number of $N$ terms in the quadrature formula when the nodes of the quadrature formula are located near the saddle point $[7,8]$. However, this contour considerably moves away from the contour of the stationary phase at large $|z|$. One can expect that the advantages of the contour coinciding with the asymptotic behavior of the exact contour of the stationary phase as $\operatorname{Re} z \rightarrow-\infty$ should be manifested for sufficiently large values of $N$. We will try to find out at what value of $N^{\star}$ occurs the "change of the mode," i.e., the use of the contour coinciding with the asymptotic behavior of the contour of a stationary phase becomes more efficient.

The paper is organized as follows. In Sec. II, we start with a review of the basic expressions relating to the choice of the integration contour in the MB integrals, according to the method proposed in Ref. [7]. In Sec. III, we explain how a contour that coincides with the asymptotic behavior of the stationary phase contour is constructed. Here, we confine ourselves to integrals with finite asymptotic behavior of the stationary phase contour as $\operatorname{Re} z \rightarrow-\infty$. We present the example of the application of constructed contour in the numerical calculation of well-known integral $I(s)$ whose value is known exactly. The case of the implementation of the $\mathrm{MB}$ integrals for evaluation of the $F_{3}\left(x_{\mathrm{B}}\right)$ structure function, where $x_{\mathrm{B}}$ is the Bjorken variable, is given in Sec. IV. We also investigate the applicability of the asymptotic stationary phase integration contour if the $Q^{2}$ dependence of the $F_{3}$ structure function is taken into account. Numerical estimates of the relative accuracy of the reconstruction of the functions considered above using different contours are given in Sec. V. Summarizing comments are presented in Sec. VI. In the Appendix, using the previously considered integral $I(s)$, we perform an additional study for the contour with finite asymptotic as $\operatorname{Re} z \rightarrow+\infty$.

\section{CONSTRUCTION OF THE $C_{K}$ INTEGRATION CONTOUR}

Let us begin with the review of the main relations for creation of the effective contour proposed by Kosower [7] based on the saddle-point method of the integrand in Eq. (1). We use this contour, which we recall is denoted by $C_{K}$, for comparison of the efficiency of the numerical evaluation of the integral (1) for different contours.

Following the saddle-point method one can rewrite expression (1) as

$$
f(x)=\frac{1}{\pi} \int_{C^{\prime}} \operatorname{Re}[-i d z F(z)],
$$

where $F(z) \equiv x^{-z} \tilde{f}(z), C^{\prime}$ means the contour running from the saddle point $c_{0}$, where $F^{\prime}\left(c_{0}\right)=0$ (see Ref. [7] for additional details). The complex variable $z$ is parametrized as follows:

$$
z(t)=x(t)+i y(t) \quad(x, y \text { real })
$$

with the conditions $x\left(t_{0}=0\right)=c_{0}$ and $y\left(t_{0}\right)=0$. Then, the expansion of $F(z(t))$ in a series around the saddle point looks like

$$
\begin{gathered}
F(z(t)) \sim F\left(c_{0}\right)-\frac{F^{\prime \prime}\left(c_{0}\right)}{2} t^{2}+\frac{1}{6}\left[-i F^{(3)}\left(c_{0}\right)\right. \\
\left.+3 i F^{\prime \prime}\left(c_{0}\right) x^{\prime \prime}(0)\right] t^{3}+\cdots .
\end{gathered}
$$

The requirement that the imaginary part of the function is equal to zero

$$
\operatorname{Im} F(z(t))=0
$$

in any order of the $F$-expansion (4) determines the exact stationary-phase contour. 
According to Eq. (5), to order $\mathcal{O}\left(t^{3}\right)$ the contour is written as

$$
z(t)=c_{0}+i t+\frac{c_{3}}{2} t^{2}
$$

where the coefficient $c_{3}=F^{(3)}\left(c_{0}\right) /\left[3 F^{\prime \prime}\left(c_{0}\right)\right]$. Hence, to this order we may write the integral (2) in the form

$$
f(x)=\frac{1}{\pi} \int_{0}^{\infty} \operatorname{Re}\left[\left(1-i c_{3} t\right) F(z(t))\right] d t
$$

with the use of the quadratic integration contour $C_{K}$ given by Eq. (6).

Next, putting $t=c_{2} \sqrt{u}$, one can introduce a new variable $u$, where $c_{2}=\sqrt{2 F\left(c_{0}\right) / F^{\prime \prime}\left(c_{0}\right)}$, and the integration over $u$ can be presented as

$$
f(x)=\int_{0}^{\infty} \frac{d u}{\sqrt{u}} e^{-u} H_{1}(u),
$$

where the function $H_{1}(u)$ up to order $\mathcal{O}(u)$ has the form

$$
H_{1}(u)=\frac{c_{2}}{2 \pi} \operatorname{Re}\left[e^{u}\left(1-i c_{3} c_{2} \sqrt{u}\right) F(z(u))\right]
$$

and the contour integration $C_{K}$ in terms of $u$ reads as

$$
z(u)=c_{0}+i c_{2} \sqrt{u}+\frac{c_{3}}{2} c_{2}^{2} u .
$$

The function $H_{1}(u)$ up to order $\mathcal{O}\left(u^{2}\right)$ looks like

$$
\bar{H}_{1}(u)=\frac{c_{2}}{2 \pi} \operatorname{Re}\left[e^{u}\left(1-i c_{3} c_{2} \sqrt{u}-4 i c_{6} c_{2}^{3} u^{3 / 2}\right) F(\bar{z})\right],
$$

and the integration contour is defined by the expression

$$
\bar{z}(u)=c_{0}+i c_{2} \sqrt{u}+\frac{c_{3}}{2} c_{2}^{2} u+c_{6} c_{2}^{4} u^{2}
$$

with the coefficients $c_{4}=c_{3} F^{(4)}\left(c_{0}\right) /\left[12 F^{\prime \prime}\left(c_{0}\right)\right]$, $c_{5}=F^{(5)}\left(c_{0}\right) /\left[120 F^{\prime \prime}\left(c_{0}\right)\right]$, and $c_{6}=\left(c_{4}-c_{5}-\frac{3}{8} c_{3}^{3}\right)$. We designate this contour by $\bar{C}_{K}$. It is obvious that if $c_{6}=0$, then expression (11) turns into Eq. (9).

Finally, one can see that the prefactor in front of the $H_{1}$ function in Eq. (8) corresponds exactly to the weight function for the generalized Laguerre polynomials $L_{n}^{(-1 / 2)}(u)$. Therefore, one can expect that the application of the generalized Gauss-Laguerre quadrature formula (see, e.g., Ref. [9])

$$
\int_{0}^{\infty} \frac{d u}{\sqrt{u}} e^{-u} H_{1}(u) \simeq \sum_{j=1}^{N} w_{j} H_{1}\left(u_{j}\right)
$$

can give a fast numerical evaluation of the integral (8) in the lower orders of approximation. This was the key achievement of the method proposed by Kosower [7]. Indeed, already the first approximation $(N=1)$ gives relative accuracy of the parton distributions reconstruction about a few percent (see, e.g., the table in Ref. [7]). However, the number of points $N$ required for evaluating the integral (8) using Eq. (13) with desirable accuracy depends on the integrand function and remains the subject of a numerical study.

\section{EXAMPLE FOR CONSTRUCTION OF ASYMPTOTIC STATIONARY PHASE CONTOUR}

To get a more detailed idea about the construction of the asymptotic stationary phase integration contour, we begin with a simple illustrative example of the MB integral, which can arise in Feynman diagrams. The asymptotics of the stationary phase contour in this case tends to a finite limit, just as in the case that will be considered below for the $F_{3}$ structure function.

Let us consider the integral [10]

$$
I(s)=\frac{1}{2 \pi i} \int_{\delta-i \infty}^{\delta+i \infty} d z(-s)^{-z} \frac{\Gamma^{3}(-z) \Gamma(1+z)}{\Gamma(-2 z) \Gamma(1-z) \Gamma(2+z)}
$$

with the fundamental strip $\delta \in(-1,0)$ in the region $0<-s<4$. This integral can be evaluated analytically with the following result: $I(s)=-s$.

We present the integrand as

$$
\Phi(z) \equiv e^{z \omega} \frac{\Gamma^{3}(-z) \Gamma(1+z)}{\Gamma(-2 z) \Gamma(1-z) \Gamma(2+z)},
$$

where $\omega=-\ln (-s)$, and using known asymptotic expressions $\Gamma(z) \sim \sqrt{2 \pi} e^{-z} z^{z-1 / 2}, z \rightarrow \infty$, and

$$
\lim _{z \rightarrow \infty} \frac{\Gamma(a+z)}{\Gamma(z) z^{a}}=1, \quad|\arg z|<\pi-\epsilon,
$$

we get the integrand asymptotic behavior

$$
\begin{aligned}
\Phi(z) & \sim|z|^{-5 / 2} \exp \left[(\omega+\ln 4) z+i \frac{3}{2} \pi \operatorname{sign}(y)-i \frac{5}{2} \arg z\right], \\
z & =x+i y .
\end{aligned}
$$

Whence from the zero phase condition $\operatorname{Im} \ln [\Phi(z)]=0$, we obtain that $\arg z=2\left[(\omega+\ln 4) y+\frac{3}{2} \pi \operatorname{sign}(y)\right] / 5$ and the asymptotic behavior of the zero-phase contour is determined by the equation

$$
x_{a s}(y)=y \operatorname{ctg}\left[\frac{2}{5}\left((\omega+\ln 4) y+\frac{3}{2} \pi \operatorname{sign}(y)\right)\right] .
$$


From this equation it follows that in the asymptotic region as $\operatorname{Re} z \rightarrow-\infty$ the zero-phase contour, which is a contour of stationary phase $C_{s t}$, tends to lines parallel to the real axis

$$
y_{a s}=\frac{\pi}{\omega+\ln 4} \operatorname{sign}(y) .
$$

Thus, the asymptotic stationary phase contour $C_{a s}$ in the complex $z$ plane takes the form

$$
z_{a s}(y)=x_{a s}(y)+i y .
$$

Using this contour, we can represent the original integral (14) as

$$
I(s)=\int_{0}^{\left|y_{a s}\right|} d y H_{2}(y),
$$

where the function $\mathrm{H}_{2}(y)$ is given by

$$
H_{2}(y)=\operatorname{Re}\left[\left(1-i \frac{d x_{a s}(y)}{d y}\right) \Phi\left(z_{a s}(y)\right)\right] / \pi,
$$

and, finally, we have

$$
\int_{0}^{\left|y_{a s}\right|} d y H_{2}(y) \simeq \frac{\left|y_{a s}\right|}{2} \sum_{j=1}^{N} w_{j} H_{2}\left(y_{j}\right),
$$

where $y_{j}=\frac{\left|y_{a s}\right|}{2}\left(x_{j}+1\right), x_{j}$ are the roots of the Legendre polynomials $P_{n}(x)$ with normalization $P_{n}(1)=1$, and weight coefficients $w_{j}=\frac{2}{\left(1-x_{j}^{2}\right)\left[P_{n}^{\prime}\left(x_{j}\right)\right]^{2}}$.

Note that in practical calculations of the integral (21), it is advisable to shift the asymptotic contour (20) parallel to the real axis to the saddle point $c_{0}$. Then, instead of Eq. (20), we get

$$
z_{a s}(y)=x_{a s}(y)+\Delta c_{0}+i y,
$$

where $\Delta c_{0}=c_{0}-c_{a s}, c_{a s} \equiv x_{a s}(y=0)$. For the case under consideration, in accordance with Eq. (18), $x_{a s}(y=0)=0$.

Comparing the shape of the contours, we also consider the exact contour of the stationary phase, which can be found from the differential equation

$$
\frac{d x}{d y}=\frac{\operatorname{Re}\left\{\partial_{z} \ln [\Phi(z)]\right\}^{*}}{\operatorname{Im}\left\{\partial_{z} \ln [\Phi(z)]\right\}^{*}}
$$

with condition $x(0)=c_{0}$. (The designation " $*$ " means the complex conjugation.) In the case under consideration, we used $x$ and $y$, since the solution $x=x(y)$ is uniquely determined. In the general case, the equation for the exact stationary-phase contour is written in the parametric form using Eq. (3); see Ref. [2] for more details.

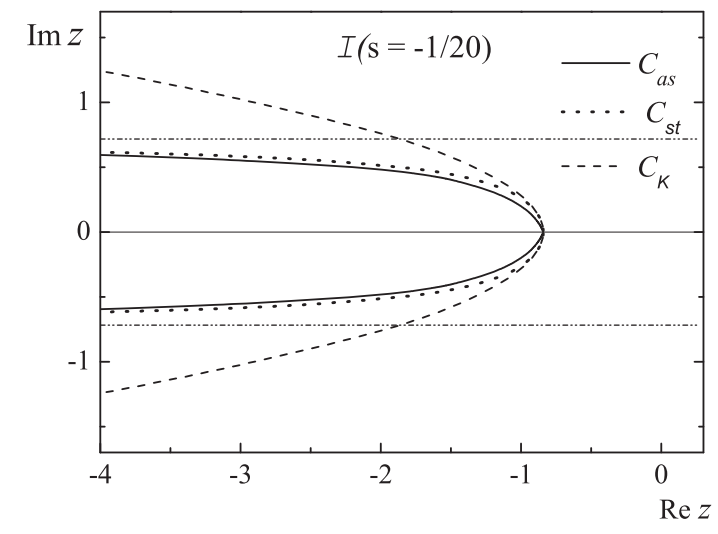

FIG. 1. The efficient contours for the MB integral (14). The solid, dotted, and dashed curves indicate the contours $C_{a s}, C_{s t}$, and $C_{K}$, respectively. Horizontal lines show the asymptotics (19).

Figure 1 shows the shape of the contours for the integral $I(s)$ given by Eq. (14) for a fixed value of $-s=1 / 20$. The solid curve represents the asymptotic stationary phase contour $C_{a s}$ determined by Eq. (24), the dotted curve corresponds to the exact contour of the stationary phase $C_{s t}$ calculated by Eq. (25), and the dashed curve is the contour $C_{K}$ corresponding to Eq. (6). The dash-dot-dotted horizontal lines denote the asymptotic limit of the stationary phase contour determined by Eq. (19). The main contribution to the integral $I(s)$ is given by a region near the saddle point $c_{0}$, where the curves are very close to each other. It can be found that the contours $C_{s t}$ and $C_{a s}$ are close also in the asymptotic region $\operatorname{Re} z \rightarrow-\infty$, but the contours $C_{K}$ and $\bar{C}_{K}$ quickly move away from the contour $C_{s t}$ (see Ref. [8] for details). Note that for the considered value of $s$, the coefficient combination $c_{6} c_{2}^{4}$ in Eq. (12) is a small negative number and the contour corresponding to Eq. (12) practically coincides with the contour $C_{K}$. Therefore, we do not plot the contour $\bar{C}_{K}$ in Fig. 1 . The influence of the coefficient $c_{6}$ for the other region of $-s>4$ is shown in the Appendix.

\section{ASYMPTOTIC STATIONARY PHASE INTEGRATION CONTOUR FOR THE $F_{3}$ STRUCTURE FUNCTION}

The reconstruction of the DIS structure function $F_{3}$ based on its Mellin moments can be performed using the inverse Mellin transformation (1), which reduces to the MB integral. For an optimal calculation of this integral we construct the efficient contours in exactly the same way as in the previous section for the integral $I(s)$. It should be noted that the accuracy of calculations of the structure function values can be limited to $4-5$ decimal places, which corresponds to the accuracy of the experimental data. At the same time, the method of calculating the $F_{3}$ structure function using the asymptotic stationary phase contour makes it easy to obtain the $F_{3}$ values with high accuracy. 


\section{A. Contour of integration}

Let us consider the following parametrization of the $F_{3}$ structure function [11]

$$
x_{\mathrm{B}} F_{3}\left(x_{\mathrm{B}}\right)=A x_{\mathrm{B}}^{\alpha}\left(1-x_{\mathrm{B}}\right)^{\beta}\left(1+\gamma x_{\mathrm{B}}\right)
$$

with values of the parameters found in Ref. [12].

We can write the Mellin moments of the structure function via the $\Gamma$ functions,

$$
\begin{aligned}
M_{3}(z) & =\int_{0}^{1} d x x^{z-1} x F_{3}(x) \\
& =A\left[\frac{\Gamma(\beta+1) \Gamma(\alpha+z)}{\Gamma(\alpha+\beta+1+z)}+\gamma \frac{\Gamma(\beta+1) \Gamma(\alpha+1+z)}{\Gamma(\alpha+\beta+2+z)}\right],
\end{aligned}
$$

and present the structure function in the form

$$
x_{\mathrm{B}} F_{3}\left(x_{\mathrm{B}}\right)=\frac{1}{2 \pi i} \int_{C} d z x_{\mathrm{B}}^{-z} M_{3}(z) .
$$

Introducing the notation

$$
\Phi(z)=e^{\omega_{\mathrm{B}} z} M_{3}(z)
$$

where $\omega_{\mathrm{B}} \equiv-\ln \left(x_{\mathrm{B}}\right)$, and using the relation (16) one can get the asymptotic behavior $\Phi(z)$ as $z \rightarrow \infty$

$$
\Phi(z) \sim e^{\omega_{\mathrm{B}} z} A \Gamma(\beta+1) \frac{1+\gamma}{z^{\beta+1}} .
$$

Calculating the argument of the $\Phi$ function and equating its imaginary part to zero, we arrive at the equation

$$
\omega_{\mathrm{B}} y-(\beta+1) \operatorname{arctg} \frac{y}{x}=0 .
$$

From this equation it follows that as $z$ tends to infinity, the argument $z$ tends to $\pm \pi$ and we get the asymptotic behavior

$$
x_{a s}^{\mathrm{DIS}}(y)=y \operatorname{ctg}\left(\frac{\omega_{\mathrm{B}} y}{\beta+1}\right) .
$$

Hence, in the asymptotic region the contour $C_{s t}(\operatorname{Re} z \rightarrow-\infty)$ tends to the finite limit

$$
y_{a s}^{\mathrm{DIS}}=\frac{(\beta+1) \pi}{\omega_{\mathrm{B}}} \operatorname{sign}(y) .
$$

As a result, the asymptotics of the stationary phase contour are parallel to the real axis. Note that the right-hand side of Eq. (33) depends on the Bjorken variable $x_{\mathrm{B}}$ and the parameter $\beta$ which relates to the shape of the structure function at large $x_{\mathrm{B}}$ values. There is no dependence on the parameters $\alpha$ and $\gamma$ contained in Eq. (26). With the

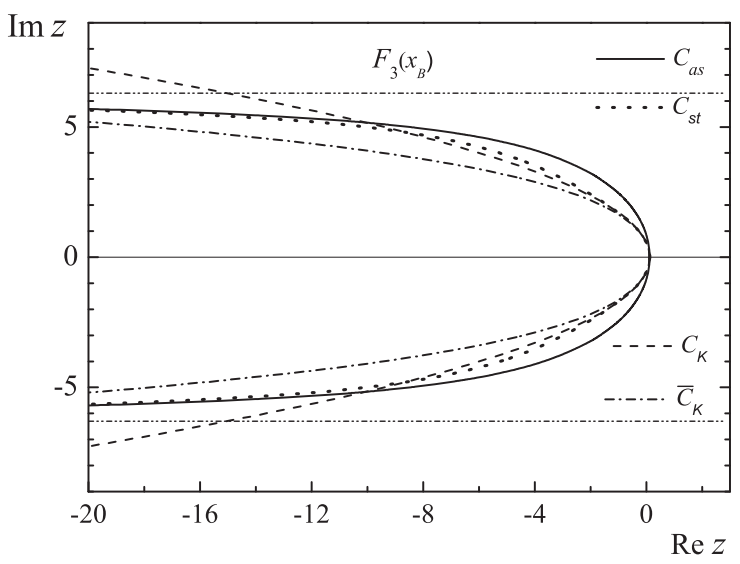

FIG. 2. The comparison of the efficient contours for the $F_{3}$ structure function at $x_{\mathrm{B}}=0.1$. The notations are the same as in Fig. 1. The contour $\bar{C}_{K}$ is shown as the dash-dotted curve. The horizontal lines correspond to the asymptotics (33).

growth of $x_{\mathrm{B}}$ and $\beta$, the width of the corridor between the asymptotic limits increases. In accordance with expression (24), we obtain that the asymptotic stationary phase contour is determined by the expression

$$
z_{a s}^{\mathrm{DIS}}=y \operatorname{ctg}\left(\frac{\omega_{\mathrm{B}} y}{\beta+1}\right)+c_{0}-\frac{\beta+1}{\omega_{\mathrm{B}}}+i y .
$$

To calculate the $F_{3}$-structure function numerically, we can use the expression (21), which is now read as

$$
x_{\mathrm{B}} F_{3}\left(x_{\mathrm{B}}\right)=\int_{0}^{\left|y_{a s}^{\mathrm{DIS}}\right|} d y H_{2}^{\mathrm{DIS}}(y),
$$

and also Eqs. (22), (23), and (29), replacing $y_{a s} \Rightarrow y_{a s}^{\text {DIS }}$ and $z_{a s} \Rightarrow z_{a s}^{\mathrm{DIS}}$.

Figure 2 shows the shape of a set of contours $C_{a s}$ (solid curve), $C_{s t}$ (dotted curve), $C_{K}$ (dashed curve), and $\bar{C}_{K}$ (dash-dotted curve) for a fixed value of $x_{\mathrm{B}}=0.1$. In the calculation, we used the parameter values in Eq. (26) that were found in Ref. [12] at fixed $Q^{2}=Q_{0}^{2}=3 \mathrm{GeV}^{2}$.

One can see that, qualitatively, the behavior of the curves in Fig. 2 is similar to the behavior in Fig. 1. There is some difference between the shapes of the contours $C_{s t}$ and $C_{a s}$ in the vicinity of the saddle point. However, in the limit $\operatorname{Re} z \rightarrow-\infty$ the contours $C_{s t}$ and $C_{a s}$ coincide, while the contours $C_{K}$ and $\bar{C}_{K}$ move away from the contour $C_{s t}$ in this limit.

\section{B. $Q^{2}$ evolution and the contour of integration}

Let us discuss the change of the behavior of the contour $C_{a s}$ with a change of the momentum transfer squared $Q^{2}$.

The perturbative $Q^{2}$ evolution of the Mellin moments is well known (see, e.g., Refs. [4,13]), and in the nonsinglet case in the leading order (LO) is given by the formula 


$$
\begin{aligned}
M_{3}\left(z, Q^{2}\right)= & M_{3}\left(z, Q_{0}^{2}\right) \exp \left\{\Delta\left(Q^{2}\right)\right. \\
& \left.\times\left[\frac{1}{2(z+1)(z+2)}+\frac{3}{4}-\gamma-\psi(z+2)\right]\right\} .
\end{aligned}
$$

Here

$$
\Delta\left(Q^{2}\right) \equiv \frac{16}{33-2 n_{f}} \ln \left[\frac{\alpha_{s}^{\mathrm{LO}}\left(Q_{0}^{2}\right)}{\alpha_{s}^{\mathrm{LO}}\left(Q^{2}\right)}\right]
$$

$\gamma$ is the Euler constant, $\psi$ is the usual logarithmic derivative of the $\Gamma$ function, $n_{f}$ is the number of active flavors, and $\alpha_{s}^{\mathrm{LO}}$ is the LO running coupling.

Finding the asymptotic behavior of the integrand $\Phi\left(z, Q^{2}\right) \equiv x_{\mathrm{B}}^{-z} M_{3}\left(z, Q^{2}\right)$ as $|z| \rightarrow \infty$, analogously to how it was made before for a fixed of value $Q^{2}$, and also taking into account an asymptotic behavior of the $\psi$ function, we obtain

$$
\Phi\left(z, Q^{2}\right) \sim \exp \left\{\omega_{\mathrm{B}} z-\left[(\beta+1)+\Delta\left(Q^{2}\right)\right] \ln z\right\} .
$$

Next, from the zero phase condition follows that $\omega_{\mathrm{B}} y-\left[(\beta+1)+\Delta\left(Q^{2}\right)\right] \arg z=0$. Therefore, the asymptotic behavior of the contour as $\operatorname{Re} z \rightarrow-\infty$ is determined by the formula

$$
x_{a S}^{\mathrm{DIS}}\left(y, Q^{2}\right)=y \operatorname{ctg}\left[\frac{\omega_{\mathrm{B}} y}{(\beta+1)+\Delta\left(Q^{2}\right)}\right] .
$$

In the asymptotic region the contour $C_{a s}$ tends to the finite limiting value

$$
y_{a s}^{\mathrm{DIS}}\left(Q^{2}\right)= \pm \frac{\left[(\beta+1)+\Delta\left(Q^{2}\right)\right] \pi}{\omega_{\mathrm{B}}} .
$$

Thus, the account of the $Q^{2}$ evolution of the Mellin moments of the $F_{3}$ structure function comes down to replacement in the expressions without evolution $\beta \Rightarrow$ $\beta+\Delta\left(Q^{2}\right)$ and using Eq. (35) $x_{a s}^{\mathrm{DIS}}(y) \Rightarrow x_{a s}^{\mathrm{DIS}}\left(y, Q^{2}\right)$ and $y_{a s}^{\text {DIS }} \Rightarrow y_{a s}^{\text {DIS }}\left(Q^{2}\right)$.

It is important to note that the expression defining the contour $C_{a s}$ in higher orders of the perturbation theory QCD will coincide with the LO expression with the replacement only of $\alpha_{s}^{\mathrm{LO}}\left(Q^{2}\right)$ in Eq. (37) by an expression for the running coupling in the corresponding order of the perturbation theory.

Figure 3 shows the influence of the $Q^{2}$ evolution on a value of the saddle point $c_{0}$ and the "asymptotic" point $c_{a s}=x_{a s}(y=0)$ in the case of the $F_{3}$ structure function. In this figure $c_{0}$ and $c_{a s}$ are shown as functions of the Bjorken variable $x_{\mathrm{B}}$ at $Q^{2}=Q_{0}^{2}=3 \mathrm{GeV}^{2}$ (solid and dashed lines for $c_{0}$ and $c_{a s}$, respectively) and $Q^{2}=100 \mathrm{GeV}^{2}$ (dotted

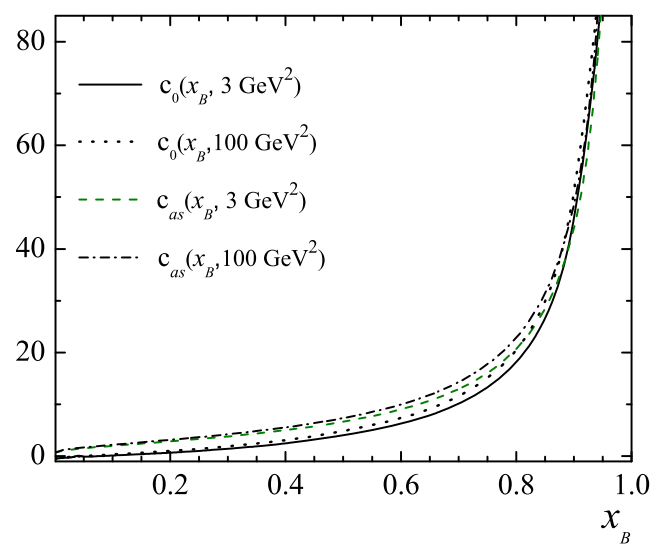

FIG. 3. The comparison of the behavior of the saddle point $c_{0}$ and the point $c_{a s}$ versus $x_{\mathrm{B}}$ at two fixed values of $Q^{2}$.

and dash-dotted lines). One can see that there is no significant difference in the behavior of the point $c_{0}$ with the changing of $Q^{2}$ as the solid and dotted curves are close to each other. The same behavior is observed also for the point $c_{a s}$. It is interesting to note a sharp increase in the values of $c_{0}$ and $c_{a s}$ if $x_{\mathrm{B}}>0.5$, as well as the convergence of all curves at large values of $x_{\mathrm{B}}$.

\section{NUMERICAL ESTIMATIONS OF ACCURACY}

In this section, by numerically calculating the MB integrals given by Eqs. (14) and (28), we investigate the question at what values of polynomials $N$ in the corresponding quadrature formula the advantages of the asymptotic contour $C_{a s}$ are manifested, in comparison with the quadratic approximation $C_{K}$ to the contour of the stationary phase.

The relative accuracy of a reconstruction is defined as usual $\varepsilon(N)=\left|\left(f_{N}-f^{\text {exact }}\right) / f^{\text {exact }}\right|$, where $f_{N}$ is the sum given by Eq. (13), when the contour $C_{K}$ is used, or Eq. (23) for the contour $C_{a s} ; f^{\text {exact }}$ is the exact value of the corresponding integral.

The relative accuracy $\varepsilon(N)$ of calculating the $I(s)$ integral depending on number of terms $N$ in the sums (13) and (23) is presented in Table I. The result is given for

TABLE I. The relative accuracy $\varepsilon(N)$ of numerical evaluation of the MB integral (14) for different values of terms $N$ in the sum (13), when the contour $C_{K}$ is used, and in the sum (23) for the contour $C_{a s}$.

\begin{tabular}{lcclcc}
\hline \hline & \multicolumn{2}{c}{$-s=1 / 20$} & & \multicolumn{2}{c}{$-s=2.0$} \\
\cline { 2 - 3 } \cline { 5 - 6 }$N$ & $C_{K}$ & $C_{a s}$ & & $C_{K}$ & $C_{a s}$ \\
\hline 16 & $1.2 \times 10^{-8}$ & $1.3 \times 10^{-7}$ & & $1.2 \times 10^{-6}$ & $6.5 \times 10^{-5}$ \\
20 & $6.7 \times 10^{-10}$ & $8.5 \times 10^{-12}$ & & $4.0 \times 10^{-6}$ & $3.9 \times 10^{-6}$ \\
30 & $1.2 \times 10^{-11}$ & $5.7 \times 10^{-14}$ & & $5.1 \times 10^{-7}$ & $1.2 \times 10^{-8}$ \\
35 & $5.8 \times 10^{-13}$ & $5.7 \times 10^{-16}$ & & $1.4 \times 10^{-7}$ & $5.8 \times 10^{-11}$ \\
\hline \hline
\end{tabular}


TABLE II. The relative accuracy $\varepsilon(N)$ of the $x_{\mathrm{B}} F_{3}\left(x_{\mathrm{B}}, Q_{0}^{2}\right)$ reconstruction for different values of terms $N$ in the sums (13) and (35), for the contours $C_{K}$ and $C_{a s}$, respectively.

\begin{tabular}{|c|c|c|c|c|c|c|}
\hline \multirow[b]{2}{*}{$N$} & \multicolumn{2}{|c|}{$x_{\mathrm{B}}=0.01$} & \multicolumn{2}{|c|}{$x_{\mathrm{B}}=0.1$} & \multicolumn{2}{|c|}{$x_{\mathrm{B}}=0.5$} \\
\hline & $C_{K}$ & $C_{a s}$ & $C_{K}$ & $C_{a s}$ & $C_{K}$ & $C_{a s}$ \\
\hline$\overline{16}$ & $2.5 \times 10^{-8}$ & $3.2 \times 10^{-7}$ & $1.3 \times 10^{-9}$ & $2.9 \times 10^{-8}$ & $1.0 \times 10^{-13}$ & $1.4 \times 10^{-11}$ \\
\hline 20 & $3.7 \times 10^{-10}$ & $4.1 \times 10^{-9}$ & $1.0 \times 10^{-10}$ & $1.6 \times 10^{-10}$ & $1.8 \times 10^{-17}$ & $4.6 \times 10^{-15}$ \\
\hline 30 & $2.7 \times 10^{-11}$ & $7.6 \times 10^{-13}$ & $5.7 \times 10^{-13}$ & $3.6 \times 10^{-16}$ & $3.8 \times 10^{-19}$ & $8.7 \times 10^{-21}$ \\
\hline 35 & $9.7 \times 10^{-13}$ & $1.5 \times 10^{-16}$ & $5.1 \times 10^{-14}$ & $1.5 \times 10^{-16}$ & $4.3 \times 10^{-20}$ & $3.6 \times 10^{-23}$ \\
\hline
\end{tabular}

$-s=1 / 20$ and $-s=2.0$. The calculations for the $F_{3}$ structure function are presented in Table II for the values $x_{B}=0.01,0.1$, and 0.5 . In doing so, we use the values of the parameters in Eq. (26) that were found in Ref. [12] at fixed $Q_{0}^{2}=3 \mathrm{GeV}^{2}$.

Tables I and II show similar results for the relative accuracy $\varepsilon(N)$. The contour $C_{K}$ works more efficiently for a small number of terms $(N<20)$ in the quadrature formulas (13) and (23), as for a large $N>20$, the result of the asymptotic contour $C_{a s}$ is more accurate by 2-3 orders of magnitude. In addition, as can be seen from Table II, the high accuracy achieved at $N^{*}=20$ exceeds the experimental accuracy by several orders of magnitude. However, as can be seen from Table I, the "regime change" for the integral $I(-s=2.0)$ occurs at a value of $\varepsilon\left(N^{*}\right) \simeq 4 \times 10^{-6}$. Such accuracy has practical importance in calculations in the quantum field theory.

Figure 4 shows the effect of the contour $Q^{2}$ evolution according to Eq. (39) for the $F_{3}$ structure function. In this figure the relative accuracy $\varepsilon(N)$ is plotted as a function of number terms $N$ in the Gauss-Legendre quadrature formula (23) at values of the Bjorken variable $x_{\mathrm{B}}=0.01$

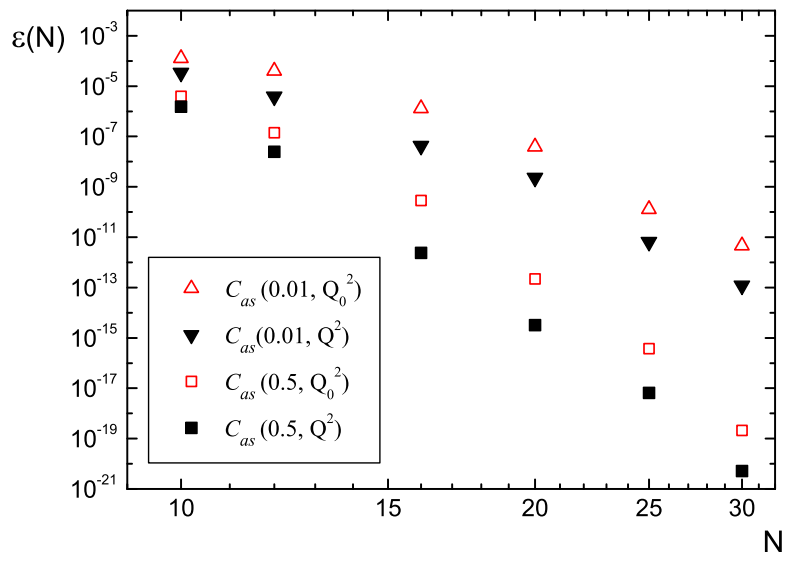

FIG. 4. The comparison of the relative accuracy $\varepsilon(N)$ of the $x_{\mathrm{B}} F_{3}\left(x_{\mathrm{B}}, Q^{2}\right)$ reconstruction versus the number of terms $N$ in the sum (23) at $x_{\mathrm{B}}=0.01$ (triangles) and $x_{\mathrm{B}}=0.5$ (squares) using the contour $C_{a s}\left(x_{\mathrm{B}}, Q_{0}^{2}\right)$ (open triangles and squares) and $C_{a s}\left(x_{\mathrm{B}}, Q^{2}\right)$ (full triangles and squares) for $Q_{0}^{2}=3 \mathrm{GeV}^{2}$ and $Q^{2}=100 \mathrm{GeV}^{2}$. (triangles) and $x_{\mathrm{B}}=0.8$ (squares) for the contours $C_{a s}\left(x_{\mathrm{B}}, Q_{0}^{2}\right) \quad$ (open triangles and squares) and $C_{a s}\left(x_{\mathrm{B}}, Q^{2}\right)$ (full triangles and squares) for $Q^{2}=Q_{0}^{2}=$ $3 \mathrm{GeV}^{2}$ and $Q^{2}=100 \mathrm{GeV}^{2}$. For the running coupling $\alpha_{s}^{\mathrm{LO}}\left(Q^{2}\right)$ we used a value $\Lambda_{\mathrm{QCD}}=363 \mathrm{MeV}$ and $n_{f}=4$ (see Ref. [12]). One can see that the contour $C_{a s}\left(x_{\mathrm{B}}, Q^{2}\right)$ yields a more exact result; however, this advantage is compensated when using the contour $C_{a s}\left(Q_{0}^{2}\right)$ if the number of terms $N$ is increased by $2-4$ units.

\section{CONCLUSIONS}

We presented a new approximation for the construction of a contour close to the contour of the stationary phase. A special case of the finite asymptotics of the stationary-phase contour in the limit $\operatorname{Re} z \rightarrow-\infty$ was considered as such an asymptotics arises in the calculation of the MB integral that represents the $F_{3}$ structure function in terms of its Mellin moments. The proposed approximation reproduces the behavior of the contour zero phase for large values of $|z|$ and has the form like Eq. (34) in the case of the DIS structure functions.

It was compared to the efficiency of application of the asymptotic stationary phase contour $C_{a s}$ and the contour $C_{K}$, determined by the saddle-point method, as described in Sec. II, in calculating the MB integrals (14) and (28). As expected, the contour $C_{K}$ turned out to be more effective for a small number of $N$ terms in the Gauss-Laguerre quadrature formula, when the nodes of the quadrature formulas are located near the saddle point $[7,8]$. The advantage of the contour $C_{a s}$ is manifested at large values of $N$. The "regime change" occurs at $N^{*} \approx 20$. For the region $20<N \leq 35$, the contour $C_{a s}$ gives a relative error of 2-3 orders of magnitude better than the contour $C_{K}$. With increasing $N$, this gap increases too.

When the $Q^{2}$ evolution of the structure function $F_{3}$ is taken into account, the efficiencies of the contours $C_{a s}\left(Q_{0}^{2}\right)$ and $C_{a s}\left(Q^{2}\right)$ were compared. Although the contour $C_{a s}\left(Q^{2}\right)$ gives a more accurate result, this advantage is compensated by using the contour $C_{a s}\left(Q_{0}^{2}\right)$ if we increase the number of terms in the quadrature formula by only $2-3$ units. The contour $C_{a s}\left(Q_{0}^{2}\right)$ can be considered as a universal one, that is, applicable for other values of $Q^{2}$. 
It should be emphasized that since the necessary accuracy is achieved when using a small number of $N$ in the quadrature formulas (13) or (21), the computer time for calculating the $F_{3}$ structure function by using Eq. (28) with the efficient contours is significantly less than if one uses the linear contours that are usually parallel to the imaginary axis, to the right of the rightmost pole in the integrand, or a straight line at an angle (see, e.g., Refs. $[4,5,14,15]$ ).

Here we have considered the $F_{3}$ structure function, which is the simplest among the DIS structure functions since it does not contain the contribution of gluons and sea quarks. The parametrization of the shape of this structure function (26) is typical and widely used in the QCD analysis of the structure functions. Our consideration can be applied to the polarized nonsinglet combination $\Delta q_{3}$, $\Delta q_{8}$ and the nonsinglet combination fragmentation function $D_{u_{v}}^{\pi^{+}}$. The choice of the efficient contour for the singlet case can be performed along the same line, but requires more complicated formulas. This is the task for a forthcoming investigation.

Our result can be useful in choosing an integration method in both the one-dimensional case and the case of few-dimensional MB integrals if it is required to achieve relative accuracy unattainable in the integration by the Monte Carlo method.

\section{ACKNOWLEDGMENTS}

This research was supported in part by the Belarusian Republican Foundation for Fundamental Research (Grant No. F16D-004), the JINR-Bulgaria Collaborative Grant, and by the Russian Foundation for Basic Research (Grant No. 16-02-00790). The authors would like to thank Professor J. Gluza for interest in this work.

\section{APPENDIX: ON NUMERICAL EVALUATION OF I $(s)$ FOR $-s>4$}

Let us turn to the MB integral (14) and discuss the efficient contours for the case $-s>4$. The exact result is written as follows:

$$
\begin{aligned}
I(s)= & 2 \ln (-s)-s\left(1-\sqrt{1+\frac{4}{s}}\right) \\
& +4 \ln \left[\frac{1}{2}+\frac{1}{2} \sqrt{1+\frac{4}{s}}\right], \quad-s>4 .
\end{aligned}
$$

This case is interesting for us, because the exact contour of zero phase $C_{s t}$ [see Eq. (25)] moves away from the imaginary axis to the right. The asymptotic behavior of the

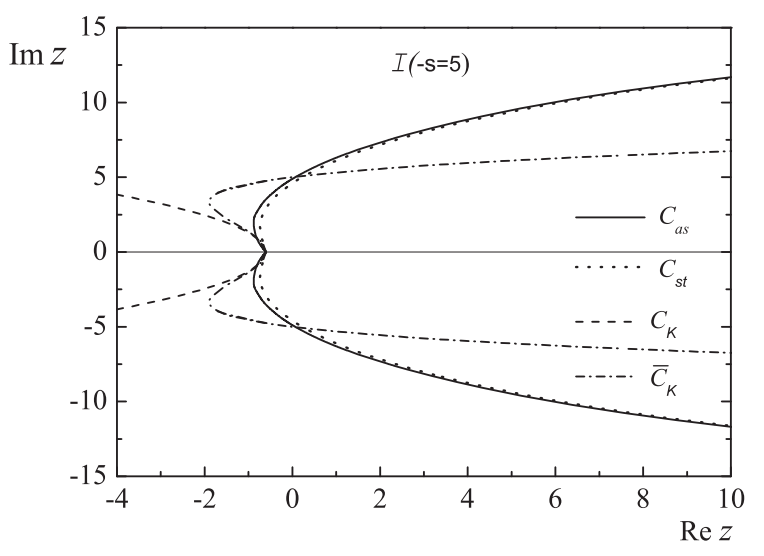

FIG. 5. The comparison of the efficient contours for the MB integral (14) in the region $-s>4$. The notations are the same as in Fig. 1.

contour $C_{s t}$ is defined by Eq. (18), as before; however, from this equation it follows that in the asymptotic region as $\operatorname{Re} z \rightarrow+\infty$ the contour tends to another limit value

$$
y_{a s}=\frac{3}{2} \frac{\pi}{|\omega+\ln 4|} \operatorname{sign}(y) .
$$

Therefore, the numerical value of the integral $I(s)$ can be found using the Gauss-Legendre quadrature formula (23) in which $y_{a s}$ is given by Eq. (A2) and the contour $C_{a s}$ by Eqs. (18) and (24).

The contours $C_{K}$ and $\bar{C}_{K}$ are defined using the same way as before, and the numerical value of the integral $I(s)$ is determined using the Gauss-Laguerre quadrature formula (13).

Figure 5 shows the shape of contours for fixed $-s=5$. The solid curve corresponds to the contour $C_{a s}$, the dotted curve is the exact contour $C_{s t}$, the dashed curve is the contour $C_{K}$, and the dash-dotted contour is the contour $\bar{C}_{K}$. From this figure it is clear that in calculating the integral $I(s)$ using the contour $C_{K}$, difficulties will arise, as this contour has an incorrect direction. Only the first few terms in the sum (13) may give a reasonable result, since the contour $C_{K}$ is close to the contour $C_{s t}$ near the saddle point $c_{0}$. Thus, for the case $-s>4$ the contour $C_{K}$ is impossible to consider as efficient. At the same time, the contour $\bar{C}_{K}$ works rather well, but applying it to achieve relative accuracy, for example, $10^{-12}$, can be problematic. The proposed contour $C_{a s}$, whose a construction is quite simple, reproduces the behavior of the exact contour $C_{s t}$ well, and the use of this contour can provide the required high relative accuracy. 
[1] H. Bateman and A. Erdelyi, Higher Transcendental Functions (McGraw-Hill, New York, 1953), Vol. 1.

[2] J. Gluza, T. Jelinski, and D. A. Kosower, Efficient evaluation of massive Mellin-Barnes integrals, Phys. Rev. D 95, 076016 (2017).

[3] M. Glück and E. Reya, Operator mixing and scaling deviations in asymptotically free field theories, Phys. Rev. D 14, 3034 (1976).

[4] M. Glück, E. Reya, and A. Vogt, Radiatively generated parton distributions for high energy collisions, Z. Phys. C 48, 471 (1990).

[5] D. Graudenz, M. Hampel, A. Vogt, and C. Berger, The Mellin transform technique for the extraction of the gluon density, Z. Phys. C 70, 77 (1996).

[6] I. Dubovyk, J. Gluza, T. Jelinski, T. Riemann, and J. Usovitsch, New prospects for the numerical calculation of Mellin-Barnes integrals in Minkowskian kinematics, Acta Phys. Pol. B 48, 995 (2017).

[7] D. A. Kosower, Evolution of parton distributions, Nucl. Phys. B506, 439 (1997).

[8] V. I. Lashkevich, A. V. Sidorov, and O. P. Solovtsova, The efficient contour of the inverse Mellin transformation for the
$F_{3}$ structure function, Nonlinear Phenom. Complex Syst. 20, 286 (2017).

[9] P. Rabinowitz and G. Weiss, Tables of abscissas and wights for numerical evaluation of integrals of the form $\int_{0}^{\infty} e^{-x} x^{n} f(x) d x$, Math. Comput. 13, 285 (1959).

[10] Recall in the paper [2] the corresponding integral is denoted by $I_{5}(s)$.

[11] In this subsection we omit the $Q^{2}$ dependence of the $F_{3}$ structure function.

[12] A. V. Sidorov and O. P. Solovtsova, The QCD analysis of the combined set for the $F_{3}$ structure function data based on the analytic approach, Mod. Phys. Lett. A 29, 1450194 (2014).

[13] A. J. Buras, Asymptotic freedom in deep inelastic processes in the leading order and beyond, Rev. Mod. Phys. 52, 199 (1980).

[14] D. de Florian, M. Epele, R. J. Hernandez-Pinto, R. Sassot, and M. Stratmann, Parton-to-kaon fragmentation revisited, Phys. Rev. D 95, 094019 (2017).

[15] E. Leader, A. V. Sidorov, and D. B. Stamenov, Determination of the fragmentation functions from an NLO QCD analysis of the HERMES data on pion multiplicities, Phys. Rev. D 93, 074026 (2016). 